\title{
Numerical investigation of a straw combustion boiler - Part I: Modelling of the thermo-chemical conversion of straw
}

\author{
Andrea Dernbecher ${ }^{1, a}$, Andreas Ortwein ${ }^{1}$ and Fouzi Tabet ${ }^{1}$ \\ ${ }^{1}$ DBFZ - Deutsches Biomasseforschungszentrum gGmbH, Torgauer Straße 116, D-04347 Leipzig, Germany
}

\begin{abstract}
In the framework of a European project, a straw combustion boiler in conjunction with an organic Rankine cycle is developed. One objective of the project is the enhancement of the combustion chamber by numerical methods. A comprehensive simulation of the combustion chamber is prepared, which contains the necessary submodels for the thermo-chemical conversion of straw and for the homogeneous gas phase reactions. Part I introduces the modelling approach for the thermal decomposition of the biomass inside the fuel bed, whereas part II deals with the simulation of the gas phase reactions in the freeboard.
\end{abstract}

\section{Introduction}

\subsection{Alternative solid fuels}

Solid biofuels are used with increasing tendency in Europe. Already in 2012, wood and solid biofuels covered $47.2 \%$ of the primary energy production by renewables in the European Union [1]. Solid biofuels are advancing, since they provide sustainable and almost $\mathrm{CO} 2$ neutral energy. Additionally, biomass can be utilized based on energy demand to compensate fluctuations in energy production from other renewables as wind or sun. However, a large biomass potential is still not yet exploited. Especially energetic utilization of agricultural residues, for example straw, can contribute to the primary energy production. But combustion of alternative solid fuels is associated with high emissions of pollutants. Straw contains significant amounts of inorganic salts, which tend to form aerosols during combustion. Aerosols are altered during their path through combustion chamber and flue pipe and form fine particles by nucleation, agglomeration, coagulation and surface reactions. The fine particles and larger particles of fly ash are entrained and carried out of the combustion chamber or precipitate on surfaces. Due to the low melting point of ash, precipitation can lead to slagging or fouling. Additionally, highly corrosive product gases can be formed from the inorganic salts, for example due to chlorine $[2,3]$.

\subsection{Biomass fuelled cogeneration technologies}

Responding to a growing interest in decentralized production of electricity from renewable resources, small and middle-scale technologies for combined heat

\footnotetext{
a Corresponding author: andrea.dernbecher@dbfz.de
}

and power (CHP) are investigated and further developed at present. Biomass fuelled technology plays an important role in this field due to the before mentioned advantages of biogenetic fuels. The organic Rankine cycle (ORC) is of interest for the combination with biomass combustion, as it is capable to use low temperature levels for electricity production. Thermal energy of the flue gas is transferred to an organic working fluid, which powers a tubine. The organic working fluid has a lower boiling point than water and therefore provides higher efficiency at moderate temperatures. This attribute allows a modular operation of ORCs with full or partial load at reasonable efficiency. Additionally, ORCs are robust to corrosive flue gases due to a heat exchanger between combustion chamber an turbine. As ORCs are operated at low temperature and pressure, less personnel is needed to supervise the process [4]. A flow scheme for a typical ORC is shown in figure 1.

An organic Rankine cycle in conjunction with straw combustion is developed in the framework of a European project. A straw combustion boiler with approximately $1 \mathrm{MW}$ power output is investigated and optimized. The boiler is used to gain thermal energy from whole bales of straw and can be operated with an additional ORC to produce electricity. For the optimization of the combustion chamber with regard to efficiency increase and emission reduction, simulations with computational fluid dynamics (CFD) are conducted. 


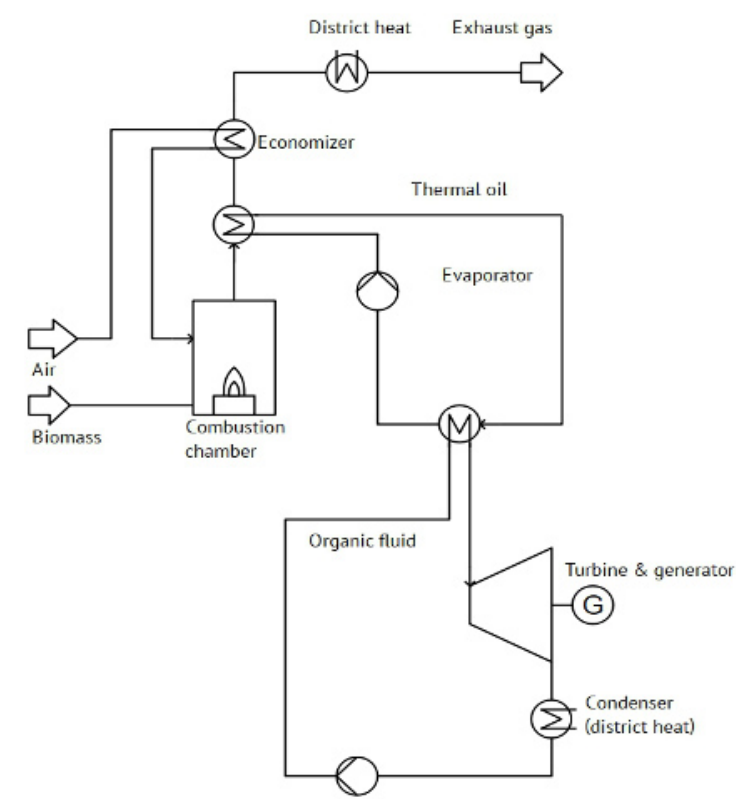

Figure 1. Flow scheme for a typical organic Rankine cycle (ORC) [5]

\subsection{CFD-Simulations for optimization of biomass combustion}

A comprehensive CFD simulation has to cover all processes inside the combustion chamber. The turbulent reactive flow in the freeboard and the thermo-chemical conversion of biomass in the fuel bed are crucial for the simulation. Additional models can be used to describe other processes, for example pollutant formation [6]. For the numerical investigation of the straw fired boiler, the bed model is particularly important, as whole bales of straw are burned inside the combustion chamber. They take a large space inside the firing chamber and influence the combustion process. Due to the transient character of the batch process, the converting biomass changes the gas flow inside the combustion chamber.

This study is divided into two parts. The first part deals with the development of a suitable bed model for straw combustion. This model contains all processes to describe thermo-chemical conversion of straw. A single particle of biomass is simulated as a test case to enhance the performance of the model and improve the sub-models.

In parallel, project partners from $\mathrm{AGH}$ in Krakow are working on the simulation of the homogeneous gas phase reactions. Details and results of their work are introduced in part II.

\section{Methods}

A three-dimensional bed model, which can be integrated into the computational domain, is needed for the description of the whole bales of straw inside the boiler. Therefore, a bed model based on a porous medium is developed. During development, a single particle model is used as a test case. The single particle model can be used for detailed improvement of the mechanisms and sub-models. Biomass undergoes the phases heating up, drying, pyrolysis, gasification and combustion during thermo-chemical conversion in oxidizing atmosphere. All phases have to be represented in a bed model.

Simulations of this study were conducted with the open source software OpenFoam [7]. The modelling approach is based on the solver biomassGasificationFoam, which was developed for gasification of biomass [8]. The solver was adapted and further developed to describe the combustion of straw. The pyrolysis mechanism was enhanced by experimental determination of kinetic parameters for straw. Furthermore, equations for the heterogeneous combustion of char were added.

The cubic particle (edge length $3.2 \mathrm{~mm}$ ) is placed inside a box, in which the walls are heated with a constant heating rate. Air enters at the inlet at the bottom of the box and streams upwards, as it can be seen in figure 2 .

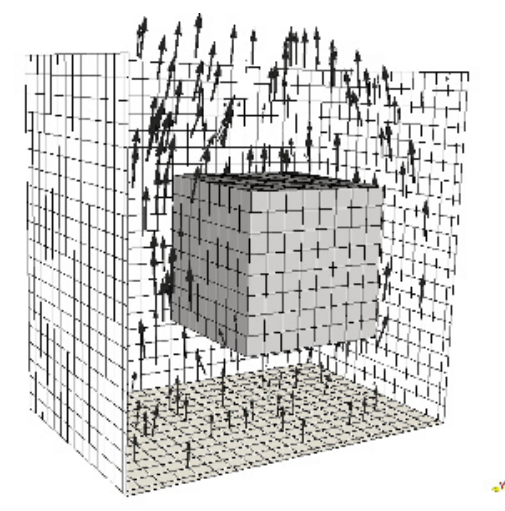

Figure 2. Geometry and mesh of the simulated straw particle [5]

Heating up of the particle is determined by heat transfer at the surface of the particle from gaseous to solid phase and heat transfer inside the particle. The temperature of gaseous and solid phase are not equal, heat transfer between the phases is described by equation (1) [9].

$$
Q=h S\left(T_{G}-T_{S}\right)
$$

In which $Q$ is the heat flow, $h$ is the heat transfer coefficient, $S$ is the surface area of the pores, $T_{G}$ is the temperature of the gaseous phase and $T_{S}$ is the temperature of the solid phase. Thermal conductivity of the solid phase is dependent from the components, which are present in the porous medium and their properties.

The initial components of biomass are humidity, cellulose, hemicellulose, lignin and ash. Humidity is consumed by drying, which is described by an Arrhenius expression in the numerical model. Therefore, evaporation of water is described by chemical reaction of the form:

$$
\text { biomass } \rightarrow \text { dry biomass }+ \text { water }
$$


Kinetics of this reaction is described by preexponential factor, activation energy and reaction order of the according Arrhenius expression (eq. (3)). These parameters were determined in an experiment with thermogravimetric analysis (TGA).

Pyrolysis is represented by three parallel reactions for the decomposition of cellulose, hemicellulose and lignin. The parameters for the kinetics of all three reactions were determined experimentally by TGA.

$$
d \alpha / d t=A \exp \left(-E_{A} / R T\right)(1-\alpha)^{n}
$$

Pre-exponetial factor $A$, activation energy $E_{A}$, reaction order $n$ and initial concentration $c$ for each component are the result of a mathematical fitting of the model equations to the measured mass loss of the biomass. $R$ and $T$ in equation (3) are the universal gas constant and the temperature in Kelvin, respectively. $\alpha$ represents the conversion of the component.

$$
\begin{gathered}
\text { cellulose } \rightarrow \text { volatiles }+ \text { char } \\
\text { hemicellulose } \rightarrow \text { volatiles }+ \text { char } \\
\text { lignin } \rightarrow \text { volatiles }+ \text { char }
\end{gathered}
$$

The kinetic parameters for drying and pyrolysis of the present study are summarized in table 1 .

Table 1. Kinetic parameters for drying and pyrolysis determined by TGA.

\begin{tabular}{|c|c|c|c|c|}
\hline & humidity & cellulose & hemicellulos & lignin \\
\hline $\mathrm{c}(\mathrm{daf})$ & - & 0.5609 & 0.2258 & 0.2133 \\
\hline $\begin{array}{c}\log \mathrm{A} / \\
\mathrm{s}^{-1}\end{array}$ & 7.738 & 12.772 & 6.172 & 5.243 \\
\hline $\begin{array}{c}\mathrm{E}_{\mathrm{A}} / \\
\mathrm{kJ} / \mathrm{mol}\end{array}$ & 63.983 & 168.22 & 86.064 & 96.193 \\
\hline $\mathrm{n}$ & 1.885 & 1.322 & 1.071 & 3.355 \\
\hline
\end{tabular}

Volatiles are composed of $\mathrm{CO}_{2}, \mathrm{CO}, \mathrm{CH}_{4}, \mathrm{H}_{2}$ and $\mathrm{H}_{2} \mathrm{O}$ in this study. The composition of product gases is based on an experimental analysis by Yang et al. [10].

Char is formed during pyrolysis and consumed by heterogeneous reactions afterwards. Char is assumed to be pure carbon for the heterogeneous reactions. Combustion of char is described by equation (7) [11].

$$
\mathrm{C}+\varphi \mathrm{O}_{2} \rightarrow 2(1-\varphi) \mathrm{CO}+(2 \varphi-1) \mathrm{CO}_{2}
$$

In which $\varphi$ is the char oxidation parameter. $\varphi$ depends on the temperature $T_{S}$ of the solid phase. Heterogeneous gasification of char is represented by equation (6) [11].

$$
\mathrm{C}+\mathrm{CO}_{2} \rightarrow 2 \mathrm{CO}
$$

Homogeneous gas phase reactions are not activated for the simulations for this study, as they are discussed in depth in part II of this paper as part of the gas phase simulation.

\section{Results and Discussion}

In Figure 3 the resulting normalized mass of the simulated single straw particle is shown.
Simulation has been conducted with a heating rate of $10 \mathrm{~K} / \mathrm{min}$.

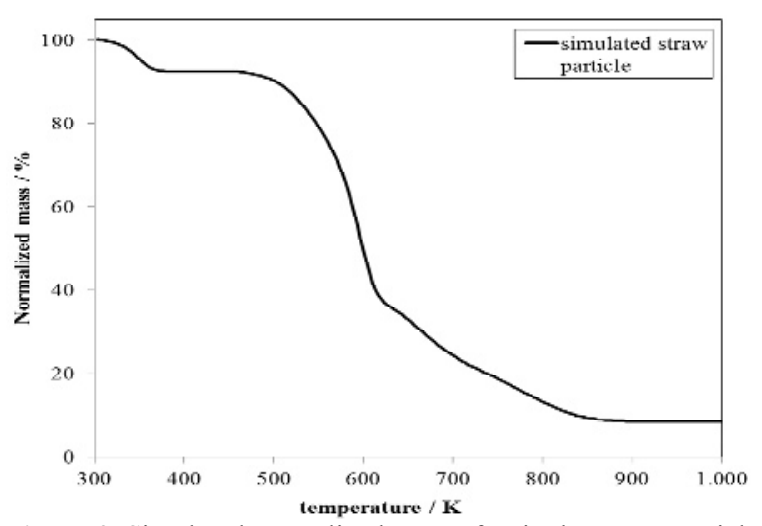

Figure 3. Simulated normalized mass of a singles straw particle in oxidizing atmosphere, heating rate $10 \mathrm{~K} / \mathrm{min}$

In the region between $300 \mathrm{~K}$ and $400 \mathrm{~K}$, drying occurs. The mass loss due to evaporation of water can clearly be identified in the graph. Starting at $450 \mathrm{~K}$, pyrolysis with decomposition of hemicellulose, cellulose and lignin occurs. Additionally to pyrolysis, heterogeneous gasification and combustion of charstart at approximately $650 \mathrm{~K}$. and increase the mass loss. After thermo-chemical conversion of the biomass particle, ash remains.

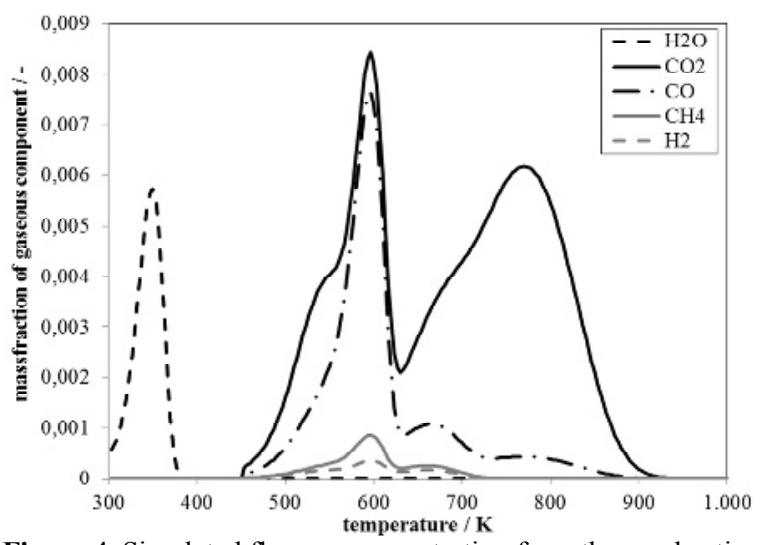

Figure 4. Simulated flue gas concentration from the combustion of a single straw particle

Concentration of the flue gases, which are formed during combustion, is shown in figure 4 . The processes of thermo-chemical conversion of biomass can be clearly identified in the curves. Water vapour has the highest concentration in the region between $300 \mathrm{~K}$ and $400 \mathrm{~K}$, where drying occurs. Pyrolysis generates large amounts of $\mathrm{CO}$ and $\mathrm{CO}_{2}$ between $450 \mathrm{~K}$ and $600 \mathrm{~K}$. Combustion of char contributes to the concentration of $\mathrm{CO}_{2}$ at temperatures above $600 \mathrm{~K}$. In comparison to $C O$ and $\mathrm{CO}_{2}$, mass fractions of methane, hydrogen and water vapour are less significant.

As different concentrations of product gases can be assigned to the decomposition of cellulose, hemicellulose and lignin and as the decomposition of these components depends on temperature, a distribution of product gas release can be observed during heating of the particle. The solver is capable of simulating a thermally thick 
biomass particle with a temperature gradient inside the particle. The resulting temperature distribution inside the simulated particle of the present study can be seen in figure 5. Due to the small size of the particle and the slow heating rate of $10 \mathrm{~K} / \mathrm{min}$, only a slight temperature gradient can be observed. In a scaled up bed model, local temperature differences will be more influential. The proposed modelling approach is capable of describing local differences in temperature and product gas composition.

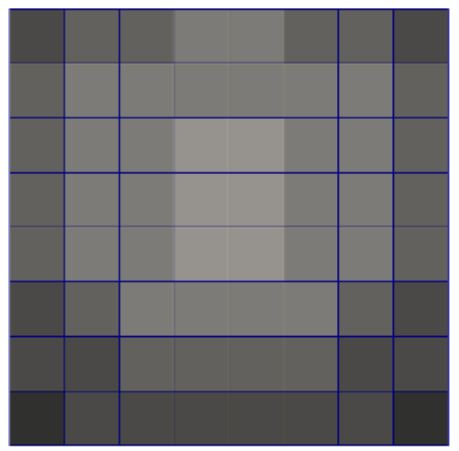

Ts

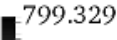

799.327

799.325

799.323

$\equiv 799.321$

Figure 5. Simulated temperature of the solid phase of the straw particle after 3000 seconds (intersection of the particle)

\section{Conclusions}

A modelling approach for the thermo-chemical conversion of straw was presented in this study. Drying, pyrolysis and heterogeneous reactions of the thermal decomposition of a straw particle were simulated. The solver biomassGasificationFoam forms a reliable basis for future simulations of a whole biomass bed inside a biomass combustion stove or boiler. The porous medium approach is suitable for the comprehensive simulation with the biomass inside the computational domain. Additionally, the composition of the product gases can be described in dependency of time and space.

\section{Outlooks}

Further improvement of model with regard to emission prediction, scale-up and accuracy is intended. To conduct comprehensive simulations of a straw firing boiler, the coupling with gas phase simulations, which are introduced in part II, is planned. The bed model can provide boundary and input conditions for the gas phase model. The comprehensive CFD simulation should be used to improve the combustion conditions inside the firing chamber of the straw boiler and adept it to the use in a cogeneration system.

Furthermore, the presented modelling approach can be used in the development of systems, which are used for energy generation by thermo-chemical conversion of biomass. Manufacturers of these systems can use CFD simulations for the development and design of new or for the improvement of existing firing systems with regard to emission reduction and efficiency increase.

\section{Acknowledgements}

The work presented in this study has been supported by KIC InnoEnergy SE within the KIC Innovation Project "Construction of cogeneration system with small to medium size biomass boilers" (Reference: 13_2014_IP92_BioORC).

\section{References}

1. European Union, Eurostat, Energy, transport and environment indicators, Luxembourg, (2014) doi:http://dx.doi.org/10.2785/56625

2. A. K. Christensen, M. Stenholm, H. Livbjerg, J Aerosol Sci 29, 4 (1998)

3. M. Jöller, T. Brunner, I. Obernberger, Energ Fuel 19 , 1 (2005)

4. L. Dong, H. Liu, S. Riffat, Appl Therm Eng, 29, No. 11-12, 2119-2126 (2009)

5. A. Dernbecher, F. Tabet, A. Ortwein, Proceedings of the European Combustion Meeting, P5-36, March 30-April 2, 2015, Budapest, Hungary, (2015)

6. F. Tabet, I. Gökalp, Renew Sust Energ Rev 51 11011114 (2015)

7. [online] [access: April 11th, 2016] http://www.openfoam.org/

8. K. Kwiatkowski, P.J. Zuk, M. Dudyński, K. Bajer, J Phys: Conf Ser 530 12-15 (2014)

9. K. Kwiatkowski, B. Górecki, J. Koroto, W. Gryglas, M. Dudyński, K. Bajer, Numer Heat Tr A-Appl, 64 216-234 (2013)

10. H. Yang, R. Yan, H. Chen, D. H. Lee, C. Zheng, Fuel 86 1781-1788 (2007)

11. M.A. Gómez, J. Porteiro, D. Patiño, J.L. Míguez, Fuel 117 716-732 (2014) 